\title{
Resposta imune humoral na COVID-19
}

\author{
Pablo Rafael S. Oliveira ${ }^{1}$ \\ Natália Machado Tavares ${ }^{2}$
}

1 Biólogo, mestre e doutor em Ciências pela Fundação Oswaldo Cruz (Fiocruz). Professor do Instituto de Biologia da Universidade Federal da Bahia (UFBA). Pesquisador colaborador do Centro de Integração de Dados e Conhecimentos para Saúde (Cidacs) da Fiocruz. Investiga os aspectos genéticos, imunológicos e epidemiológicos de doenças infecciosas.

2 Tem graduação em Biologia, mestrado e doutorado em Patologia, todos pela Universidade Federal da Bahia (UFBA). É pesquisadora em saúde pública da Fundação Oswaldo Cruz (Fiocruz) e atua em linhas de pesquisa sobre imunidade inata em doenças infecciosas/ inflamatórias e comorbidades.

OLIVEIRA, P. R. S.; TAVARES, N. M. Resposta imune humoral na COVID-19. In: BARRAL-NETTO, M.; BARRETO, M. L.; PINTO JUNIOR, E. P.; ARAGÃO, E. (org.). Construção de conhecimento no curso da pandemia de COVID-19: aspectos biomédicos, clínico-assistenciais, epidemiológicos e sociais. Salvador: Edufba, 2020. v. 1. https://doi. org/10.9771/9786556300443.004. 


\section{Introdução}

Diversos estudos foram conduzidos com o objetivo de explorar a resposta imunológica na COVID-19. (BAO et al., 2020; PARK; IWASAKI, 2020; WEN et al., 2020) No entanto, a magnitude e a duração das respostas de anticorpos contra o SARS-CoV-2 ainda não são bem compreendidas. Apesar de um grande número de estudos evidenciar que a infecção induz imunidade protetora, outros estudos sugerem que alguns pacientes com COVID-19 podem não apresentar quantidades detectáveis de Anticorpos Neutralizantes (nAb) e Imunoglobulina G (IgG) contra o vírus e que, mesmo em pacientes hospitalizados, a concentração plasmática de $\mathrm{nAb}$ pode ser extremamente baixa. (HUANG et al., 2020; WU et al., 2020) Recentemente, Long e demais autores (2020) observaram que indivíduos que não desenvolvem sintomas $(\mathrm{n}=37)$ da COVID-19 apresentam resposta humoral mais fraca ao SARS-CoV-2 do que aqueles sintomáticos ( $\mathrm{n}=37$ ). Nesse pequeno estudo, $40 \%$ dos assintomáticos e cerca de $13 \%$ dos sintomáticos apresentaram níveis indetectáveis de anticorpos IgG na fase convalescente inicial. Apesar disso, é importante ressaltar que baixas concentrações de nAbs podem exercer efeitos protetores. De fato, Robbiani e demais autores (2020) sugerem que 
mesmo níveis baixos de anticorpos podem ser suficientes para neutralizar o SARS-CoV-2. Considerando as limitações e os resultados divergentes dos estudos citados, são necessárias investigações adicionais sobre a indução e duração da resposta protetora de anticorpos frente à infecção pelo SARS-CoV-2.

Nesse sentido, estudos envolvendo outros coronavírus que infectam humanos podem fornecer pistas importantes. A glicoproteína spike (S) da superfície viral está presente nos coronavírus SARS-CoV-1 e SARS-CoV-2, que pertencem ao mesmo sub-gênero Sarbecovirus, compartilhando cerca de $80 \%$ de similaridade na sequência de aminoácidos. (WALLS et al., 2019) Assim, já foi relatado que um anticorpo monoclonal gerado em um indivíduo infectado pelo SARS-CoV-1 potencialmente neutraliza o SARS-CoV-2 e outros sarbecovírus. Esse estudo demonstrou que o anticorpo S309 reconhece um epítopo da proteína S altamente conservado entre os sarbecovírus. Além da atividade neutralizante, esse anticorpo parece recrutar mecanismos efetores e ter efeito sinérgico com outros anticorpos, potencializando o controle da infecção. (PINTO et al., 2020)

Outros estudos também identificaram reatividade cruzada de anticorpos contra a proteína $\mathrm{N}$ (nucleocapsídeo) do SARS-CoV-1 e SARS-CoV-2, que compartilham cerca de $90 \%$ de similaridade na sequência de aminoácidos. (GUO et al., 2020; OKBA et al., 2020) Esses estudos sugerem, então, que a exposição prévia a outros sarbecovírus pode gerar uma resposta anticórpica com algum grau de reatividade cruzada, mitigando o risco de escape viral. No entanto, ensaios para determinar a afinidade desses anticorpos indicaram que ela é muito baixa, sendo pouco provável que essa reatividade cruzada tenha alguma função protetora contra o SARS-CoV-2. (EMMENEGGER et al., 2020)

Além de apresentar grande semelhança genética com outros betacoronavírus, o SARS-CoV-2 pode causar síndrome respiratória 
grave em seres humanos, quadro clínico muito semelhante ao observado em infecções pelos vírus SARS-CoV-1 e MERS-CoV. (CHAN et al., 2020; YANG et al., 2020) Estudos mostram que, embora os títulos de Imunoglobulina M (IgM) e IgG contra SARS-CoV-1 ou MERS-CoV aumentem substancialmente durante as primeiras semanas após o início dos sintomas, as concentrações plasmáticas de IgM diminuem gradualmente durante o primeiro mês de infecção, o que não ocorre em relação às concentrações plasmáticas de IgG. (HSUEH et al., 2004; SPANAKIS et al., 2014) Adicionalmente, foi observado que pacientes recuperados da doença causada pelo MERS-CoV apresentaram níveis detectáveis de IgG até um ano após o início dos sintomas. (CHOE et al., 2017) Cao e demais autores (2007) relataram que os títulos de IgG e nAb para o SARS-CoV-1 atingem as maiores concentrações no quarto mês após a infecção e que a maioria dos pacientes apresentam quantidades detectáveis desses anticorpos no plasma por pelo menos três anos.

Em relação ao SARS-CoV-2, estudos recentes observaram aumentos graduais das concentrações plasmáticas de IgM e IgG, acompanhando a evolução da infecção. (LONG et al., 2020; ZHANG et al., 2020) Nesse contexto, anticorpos IgM podem ser identificados no plasma de pacientes após três dias de infecção, com pico observado em duas ou três semanas, mantendo níveis detectáveis após um mês. (XIAO; GAO; ZHANG, 2020) De outro modo, anticorpos IgG anti-SARS-CoV-2 podem ser detectados já no quarto dia após a infecção, com pico sendo atingido, em média, após 17 dias (Figura 1). De acordo com Hou e demais autores (2020), altas concentrações de IgG foram detectadas no plasma de pacientes com COVID-19 dois meses após a infecção (período de duração do estudo). Outro estudo evidenciou que na COVID-19, a resposta de IgA sérica é mais intensa e persistente do que a resposta de IgM, podendo ser detectada na primeira semana após 
o início dos sintomas em $75 \%$ dos pacientes. Além disso, o pico da resposta de Imunoglobulina A (IgA) foi identificado entre 20 e 22 dias após o aparecimento dos sintomas e esses níveis elevados foram mantidos por mais de 40 dias. (PADOAN et al., 2020)

Figura 1 - Soroconversão dos anticorpos produzidos em indivíduos assintomáticos e sintomáticos com COVID-19
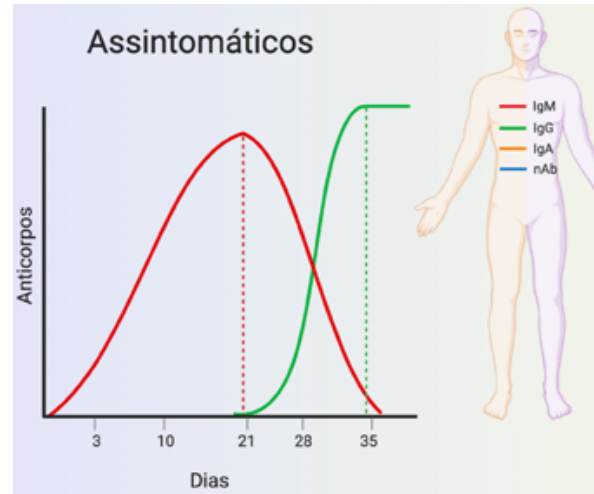

Fonte: elaborada pelos autores. ${ }^{3}$

\section{Sintomáticos}

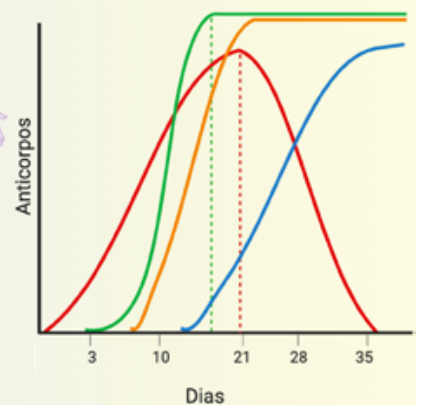

Nota: Indivíduos assintomáticos podem apresentar títulos de IgM após contato com o vírus, no entanto os títulos de IgG parecem ser mais tardios. Nos indivíduos sintomáticos, os títulos de IgM e IgG podem estar associados a gravidade da doença e o desenvolvimento clínico. Os títulos de IgA demonstram ser mais intensos do que os de lgM, sendo observado até mais de 40 dias após o início dos sintomas. Os nAbs (neutralizantes) apresentam títulos proporcionais ao lgG, surgindo a partir da segunda semana após os sintomas. Em vermelho, a curva da imunoglobulina $M(\lg M)$, em verde, a imunoglobulina $G(\lg G)$, em amarelo, a imunoglobulina $A(\lg A)$ e, em azul, os anticorpos neutralizantes (nAb).

3 Design gráfico de Sara Nunes. 


\section{Resposta de anticorpos e o curso clínico da infecção pelo SARS-GoV-2}

Embora os títulos de anticorpos sejam geralmente usados como preditores de proteção, foi observado que altos títulos de anticorpos e soroconversão precoce têm correlação com a gravidade da doença em pacientes infectados com o vírus SARS-CoV-1. (LEE et al., 2020) Além disso, foi demonstrado que as concentrações plasmáticas de IgM e IgG são associadas ao curso clínico e à gravidade da doença causada pelo MERS-CoV. (ALSHUKAIRI et al., 2016) Achados semelhantes já foram descritos no contexto da infecção pelo SARS-CoV-2, cuja indução da resposta anticórpica nem sempre é acompanhada de redução do RNA viral, especialmente em pacientes com quadro clínico grave. Ademais, foi observada uma correlação positiva entre a gravidade da doença e os títulos de anticorpos, sugerindo que apresentar altos títulos de anticorpos pode ser considerado um fator de risco para evolução com quadro crítico da doença. (ZHAO et al., 2020) Diante das limitações do estudo que podem ter influenciado essa análise, investigações específicas para identificar a relevância clínica dos títulos de anticorpos contra SARS-CoV-2 e o desfecho da doença são necessários.

Em estudo recente sobre a cinética da resposta de anticorpos durante a COVID-19, Tan e demais autores (2020) observaram que a soroconversão de IgM acontece praticamente ao mesmo tempo em casos graves e não graves, mas que a soroconversão de IgG ocorre mais precocemente em casos graves. Em outro estudo, Hou e demais autores (2020) observaram que, nos indivíduos com sintomas leves, os títulos de IgG se mantiveram altos ao longo de todo o período estudado, enquanto os títulos de IgM diminuíram após a recuperação desses pacientes. Nesse mesmo estudo, foi relatado aumento das concentrações de IgM e diminuição das concentrações de anticorpos IgG anti-SARS-CoV-2 em pacientes em estado crítico. 
Esses e outros estudos indicam que a grande maioria dos indivíduos recuperados da COVID-19 rapidamente produz anticorpos com funções neutralizantes em resposta a diferentes proteínas virais. Os títulos desses anticorpos variam amplamente entre os indivíduos, provavelmente devido à carga viral e manifestações clínicas mais leves ou graves. No entanto, uma parcela sequer apresenta níveis detectáveis de anticorpos neutralizantes. Um estudo que avaliou a resposta imune humoral de 14 pacientes que foram hospitalizados com COVID-19 e se recuperaram da doença, relatou que um desses pacientes não apresentou atividade neutralizante no soro. (NI et al., 2020) A despeito da presença de IgM, esse paciente não apresentou produção detectável de IgG ou IgG1. Por outro lado, sua resposta de células T apresentava características funcionais contra o SARS-CoV-2, sugerindo que a resposta imune celular também pode ter papel decisivo no desfecho da doença.

Outro estudo identificou que indivíduos assintomáticos apresentam resposta humoral mais tardia ou não apresentam soroconversão. (YONGCHEN et al., 2020) Dos cinco indivíduos avaliados, apenas um desenvolveu resposta anticórpica (IgG) após três semanas do diagnóstico. Os demais indivíduos assintomáticos não apresentaram soroconversão até quatro semanas após o diagnóstico (Figura 1). Por outro lado, todos os pacientes hospitalizados avaliados nesse estudo apresentaram soroconversão ainda durante a internação ou durante o período de seguimento do estudo. (YONGCHEN et al., 2020) A ausência de resposta imune humoral poderia ser explicada pela eficiência da resposta imune inata local ou pela produção de IgA (CERVIA et al., 2020) nas mucosas expostas a baixas cargas virais. Esses fenômenos levariam a uma resolução precoce da infecção através de fagócitos residentes, sem indução de resposta imune adaptativa. 


\section{O papel de anticorpos neutralizantes na infecção pelo SARS-CoV-2}

De modo geral, as respostas de anticorpos contra vírus envelopados, como os coronavírus, são tipicamente induzidas com resposta primária e secundária, compostas por IgM, IgG (IgG3, IgG1) e IgA para glicoproteínas do capsídeo viral e nucleoproteínas. Nesse contexto, Amanat e demais autores (2020) identificaram que IgG3 representa o subtipo dominante de anticorpos durante a infecção pelo SARS-CoV-2. Foi observado também que os títulos de IgG no soro de pacientes com COVID-19 apresentam forte correlação com anticorpos que neutralizam a replicação viral em culturas de células (WÖLFEL et al., 2020; ZHAO et al., 2020) e que nAbs específicos para o SARS-CoV-2 são encontrados em mais de $90 \%$ dos pacientes em até duas semanas após o início dos sintomas (Figura 1). (WU et al., 2020) De modo geral, os nAbs são definidos in vitro por suas capacidades em bloquear a fusão ou a entrada do patógeno na célula-alvo. (IWASAKI; YANG, 2020)

Como a entrada do SARS-CoV-2 nas células-alvo depende da interação entre a glicoproteína viral S (spike) e o receptor celular Enzima Conversora da Angiotensina 2 (ECA2), nAbs específicos para a proteína $S$ - sobretudo, para o Domínio de Ligação ao Receptor (RBD) - podem efetivamente suprimir a disseminação do vírus. De fato, estudos prévios identificaram anticorpos eficazes na neutralização do SARS-CoV-1 (in vitro e in vivo), que reconhecem o domínio RBD da glicoproteína $S$ do vírus. (BERRY et al., 2004; TER MEULEN et al., 2006) Ou e demais autores (2020) demonstraram que um anticorpo recombinante conhecido como T62 (anti-SARS-CoV-1 S) inibe a entrada do SARS-CoV-1 em células que expressam ECA2 (293/hECA2). Recentemente, foram isolados e clonados dois nAbs (CA1 e CB6) do plasma de um paciente com COVID-19 (SHI et al., 2020), esses anticorpos apresentam forte 
capacidade neutralizante in vitro. Além disso, o anticorpo monoclonal CB6, que reconhece um epítopo no domínio RBD da glicoproteína $\mathrm{S}$ do SARS-CoV-2, foi eficaz no combate à infecção em macacos rhesus. Em estudo similar, Ju e demais autores (2020) identificaram dezenas de anticorpos capazes de neutralizar o SARS-CoV-2, cujos efeitos práticos também estão relacionados ao bloqueio da ligação do domínio RBD da glicoproteína $S$ do vírus ao receptor celular ECA2. Os autores relataram ainda que nem os anticorpos isolados anti-SARS-CoV-2 nem o plasma dos indivíduos infectados reagiram de forma cruzada com as glicoproteínas $S$ dos vírus SARS-CoV-1 ou MERS-CoV. Um estudo anterior já havia observado potencial limitado de neutralização cruzada entre soros convalescentes de SARS-CoV-1 e SARS-CoV-2, indicando que os anticorpos produzidos por indivíduos previamente recuperados de infecção por SARS-CoV-1 não teriam capacidade de proteger contra a infecção por SARS-CoV-2, ou vice-versa. (OU et al., 2020)

\section{Agravamento da COVID-19 mediado por anticorpos?}

Além da capacidade de neutralização e de outras funções protetoras, os anticorpos podem interagir com outros componentes do sistema imune, incluindo fatores do sistema complemento, fagócitos e células NK, promovendo a infecção de células-alvo e causando o agravamento de doenças. Esse tipo de resposta mediada por anticorpos, conhecida como Agravamento Dependente de Anticorpos (ADE), sigla em inglês para antibody-dependent enhancement), já foi descrita na infecção pelo vírus da dengue (KLIKS et al., 1988), além de ter sido demonstrada no contexto da infecção pelo SARS-CoV-1. (JAUME et al., 2011; YASUI et al., 2014) Na doença causada pelo SARS-CoV-1, os complexos vírus-anticorpo podem amplificar a resposta inflamatória pela ativação de células mielóides 
através de receptores Fragmento Cristalizável (Fc). (WANG et al., 2014) Além disso, a maior captação do vírus por macrófagos através do ADE pode levar ao aumento da produção de Fator de Necrose Tumoral (TNF) e Interleucina 6 (IL-6). Foi demonstrado em camundongos infectados com SARS-CoV-1 que a ocorrência de ADE é associada à diminuição dos níveis de citocinas anti-inflamatórias IL-10 e Fator de transformação do crescimento beta (TGF $\beta$ ) e ao aumento dos níveis das quimiocinas pró-inflamatórias CCL2 e CCL3. (YASUI et al., 2008)

Dessa forma, considerando todos esses aspectos, será importante investigar a ocorrência de $\mathrm{ADE}$ na COVID-19. Os resultados desses estudos serão determinantes para o direcionamento de estratégias terapêuticas ou profiláticas com base em anticorpos neutralizantes monoclonais e plasma convalescente, além de impactar na seleção de antígenos para vacinas contra o SARS-CoV-2.

\section{Referências}

ALSHUKAIRI, A. N. et al. Antibody response and disease severity in healthcare worker MERS survivors. Emerging Infectious Diseases, Atlanta, v. 22, n. 6,

p. 1113-1115, 2016. DOI: 10.3201/eid2206.160010. Disponível em: https://wwwnc. cdc.gov/eid/article/22/6/16-0010_article. Acesso em: 29 jul. 2020.

AMANAT, F. et al. A serological assay to detect SARS-CoV-2 seroconversion in humans. Nature Medicine, New York, v. 26, p. 1033-1036, 2020.

DOI: 10.1101/2020.03.17.20037713. Disponível em: https://www.nature.com/ articles/s41591-020-0913-5. Acesso em: 29 jul. 2020.

BAO, L. et al. Lack of Reinfection in Rhesus Macaques Infected with SARS-CoV-2. BioRxiv, [s. I.], 2020. DOI: 10.1101/2020.03.13.990226. Disponível em: https://www. biorxiv.org/content/10.1101/2020.03.13.990226v2. Acesso em: 29 jul. 2020. 
BERRY, J. D. et al. Development and characterisation of neutralising monoclonal antibody to the SARS-coronavirus. Journal of Virology Methods, Amsterdam, v. 120, n. 1, p. 87-96, 2004. DOI: 10.1016/j.jviromet.2004.04.009. Disponível em: https:// www.sciencedirect.com/science/article/pii/S0166093404001272?via\%3Dihub. Acesso em: 29 jul. 2020.

CAO, W. C. et al. Disappearance of antibodies to SARS-associated coronavirus after recovery. New England Journal of Medicine, Boston, v. 357, n. 11, p. 1162-1163, 2007. DOI: 10.1056/NEJMc070348. Disponível em: https://www.nejm.org/doi/ full/10.1056/NEJMc070348. Acesso em: 29 jul. 2020.

CERVIA, C. et al. Systemic and mucosal antibody secretion specific to SARS-CoV-2 during mild versus severe COVID-19. BioRxiv, [s. I.], 2020. DOI: https://doi. org/10.1101/2020.05.21.108308. Disponível em: https://www.biorxiv.org/ content/10.1101/2020.05.21.108308v1. Acesso em: 29 jul. 2020.

CHAN, J. F. W. et al. Genomic characterization of the 2019 novel humanpathogenic coronavirus isolated from a patient with atypical pneumonia after visiting Wuhan. Emerging Microbes \& Infections, Atlanta, v. 9, n. 1, p. 221-236, 2020. DOI: 10.1080/22221751.2020.1719902. Disponível em: https://www.tandfonline. com/doi/full/10.1080/22221751.2020.1719902. Acesso em: 29 jul. 2020.

CHOE, P. G. et al. MERS-CoV antibody responses 1 year after symptom onset, South Korea, Emerging Microbes \& Infections, Atlanta, v. 23, n. 7, p. 1079-1084, 2017. DOI: 10.3201/eid2307.170310. Disponível em: https://wwwnc.cdc.gov/eid/ article/23/7/17-0310_article. Acesso em: 29 jul. 2020.

EMMENEGGER, M. et al. Population-wide evolution of SARS-CoV-2 immunity tracked by a ternary immunoassay. MedRxiv, [s. I.], 2020. DOI: https://doi.org /10.1101/2020.05.31.20118554. Disponível em: https://www.medrxiv.org/ content/10.1101/2020.05.31.20118554v2. Acesso em: 29 jul. 2020.

GUO, L. et al. Profiling Early Humoral Response to Diagnose Novel Coronavirus Disease (COVID-19). Clinical Infectious Diseases, Chicago, 2020. DOI: 10.1093/cid/ ciaa310. Disponível em: https://academic.oup.com/cid/article/71/15/778/5810754. Acesso em: 29 jul. 2020.

$\mathrm{HOU}, \mathrm{H}$. et al. Detection of IgM and IgG antibodies in patients with coronavirus disease 2019. Clinical \& Translational Immunology, London, v. 9, n. 5, p. e01136, 2020. DOI: 10.1002/cti2.1136. Disponível em: https://onlinelibrary.wiley.com/doi/ full/10.1002/cti2.1136. Acesso em: 29 jul. 2020. 
HSUEH, P. R. et al. Chronological evolution of IgM, IgA, IgG and neutralisation antibodies after infection with SARS-associated coronavirus. Clinical Microbiology and Infection, Paris, v. 10, n. 12, p. 1062-1066, 2004. DOI: 10.1111/j.1469-0 691.2004.01009.x. Disponível em: https://linkinghub.elsevier.com/retrieve/pii/ S1198743X14638477. Acesso em: 29 jul. 2020.

HUANG, A. T. et al. A systematic review of antibody mediated immunity to coronaviruses: antibody kinetics, correlates of protection, and association of antibody responses with severity of disease. MedRxiv, [s. I.], 2020.

DOI: 10.1101/2020.04.14.20065771. Disponível em: https://www.medrxiv.org/ content/10.1101/2020.04.14.20065771v1. Acesso em: 29 jul. 2020.

IWASAKI, A.; YANG, Y. The potential danger of suboptimal antibody responses in COVID-19. Nature Reviews Immunology, London, v. 20, n. 6, p. 339-341, 2020. DOI: 10.1038/s41577-020-0321-6. Disponível em: https://www.nature.com/ articles/s41577-020-0321-6. Acesso em: 29 jul. 2020.

JAUME, M. et al. Anti-Severe Acute Respiratory Syndrome Coronavirus Spike Antibodies Trigger Infection of Human Immune Cells via a pHand Cysteine Protease-Independent Fc R Pathway. Journal of Virology, Baltimore, v. 85, n. 20, p. 10582-10597, 2011. DOI: 10.1128/JVI.00671-11. Disponível em: https://jvi.asm.org/content/85/20/10582. Acesso em: 29 jul. 2020.

JU, B. et al. Human neutralizing antibodies elicited by SARS-CoV-2 infection. Nature, London, 2020. DOI: 10.1038/s41586-020-2380-z. Disponível em: https://www.nature.com/articles/s41586-020-2380-z. Acesso em: 29 jul. 2020.

KLIKS, S. C. et al. Evidence that maternal dengue antibodies are important in the development of dengue hemorrhagic fever in infants. American Journal of Tropical Medicine and Hygiene, Mclean, VA, v. 38, n. 2, p. 411-419, 1988. DOI: 10.4269/ajtmh.1988.38.411. Disponível em: http://www.ajtmh.org/content/ journals/10.4269/ajtmh.1988.38.411. Acesso em: 29 jul. 2020.

LEE, Y. L. et al. Dynamics of anti-SARS-Cov-2 IgM and IgG antibodies among COVID-19 patients. Journal of Infection, London, 2020. DOI: 10.1016/j. jinf.2020.04.019. Disponível em: https://linkinghub.elsevier.com/retrieve/pii/ S0163445320302309. Acesso em: 29 jul. 2020.

LONG, Q. X. et al. Clinical and immunological assessment of asymptomatic SARS-CoV-2 infections. Nature Medicine, New York, 2020. DOI: 10.1038/ s41591-020-0965-6. Disponível em: https://www.nature.com/articles/ s41591-020-0965-6. Acesso em: 29 jul. 2020. 
$\mathrm{NI}$, L. et al. Detection of SARS-CoV-2-Specific Humoral and Cellular Immunity in COVID-19 Convalescent Individuals. Immunity, Cambridge, v. 52, n. 6, p. 971-997, 2020. DOI: https://doi.org/10.1016/j.immuni.2020.04.023. Disponível em: https:// www.sciencedirect.com/science/article/pii/S1074761320301813. Acesso em: 29 jul. 2020.

OKBA, N. M. A. et al. Severe Acute Respiratory Syndrome Coronavirus 2-Specific Antibody Responses in Coronavirus Disease 2019 Patients. Emerging Infectious Diseases, Atlanta, v. 26, n. 7, p. 1478-1488, 2020. DOI: 10.3201/eid2607.200841. Disponível em: https://wwwnc.cdc.gov/eid/article/26/7/20-0841_article. Acesso em: 29 jul. 2020.

$\mathrm{OU}, \mathrm{X}$. et al. Characterization of spike glycoprotein of SARS-CoV-2 on virus entry and its immune cross-reactivity with SARS-CoV. Nature Communication, London, v. 11, n. 1, 2020. DOI: 10.1038/s41467-020-15562-9. Disponível em: https://www.nature.com/articles/s41467-020-15562-9. Acesso em: 29 jul. 2020.

PADOAN, A. et al. IgA-Ab response to spike glycoprotein of SARS-CoV-2 in patients with COVID-19: A longitudinal study. Clinica Chimica Acta, Amsterdam, v. 507, p. 164-166, 2020. DOI: 10.1016/j.cca.2020.04.026. Disponível em: https:// linkinghub.elsevier.com/retrieve/pii/S0009898120301819. Acesso em: 29 jul. 2020.

PARK, A.; IWASAKI, A. Type I and Type III Interferons - Induction, Signaling, Evasion, and Application to Combat COVID-19. Cell Host \& Microbe, Cambridge, v. 27, n. 6, p. 870-878, 2020. DOI: 10.1016/j.chom.2020.05.008. Disponível em: https://www.sciencedirect.com/science/article/pii/S1931312820302900. Acesso em: 29 jul. 2020.

PINTO, D. et al. Cross-neutralization of SARS-CoV-2 by a human monoclonal SARS-CoV antibody. Nature, London, v. 583, p. 290-295, 2020. Disponível em: https://www.nature.com/articles/s41586-020-2349-y. Acesso em: 29 jul. 2020.

ROBBIANI, D. F. et al. Convergent antibody responses to SARS-CoV-2 in convalescent individuals. Nature, London, 2020. DOI: 10.1038/s41586-020-2456-9. Disponível em: https://www.nature.com/articles/s41586-020-2456-9. Acesso em: 29 jul. 2020.

$\mathrm{SHI}$, R. et al. A human neutralizing antibody targets the receptor binding site of SARS-CoV-2. Nature, London, 2020. DOI: 10.1038/s41586-020-2381-y. Disponível em: https://www.nature.com/articles/s41586-020-2381-y. Acesso em: 29 jul. 2020. 
SPANANKIS, N. et al. Virological and serological analysis of a recent Middle East respiratory syndrome coronavirus infection case on a triple combination antiviral regimen. International Journal of Antimicrobial Agents, Amsterdam, v. 44, n. 6, p. 528-532, 2014. DOI: 10.1016/j.ijantimicag.2014.07.026. Disponível em: https:// www.sciencedirect.com/science/article/pii/S0924857914002787?via\%3Dihub. Acesso em: 29 jul. 2020.

TAN, W. et al. Viral Kinetics and Antibody Responses in Patients with COVID19. MedRxiv, [s. I.], 2020. DOI: https://doi.org/10.1101/2020.03.24.20042382. Disponível em: https://www.medrxiv.org/content/10.1101/2020.03.24.20042382v1. Acesso em: 29 jul. 2020.

TER MEULEN, J. et al. Human monoclonal antibody combination against SARS coronavirus: Synergy and coverage of escape mutants. PLoS Medicine, San Francisco, v. 3, n. 7, p. 1071-1079, 2006. DOI: 10.1371/journal.pmed.0030237. Disponível em: https://journals. plos.org/plosmedicine/article?id=10.1371/journal. pmed.0030237. Acesso em: 29 jul. 2020.

WALLS, A. C. et al. Unexpected Receptor Functional Mimicry Elucidates Activation of Coronavirus Fusion. Cell, Cambridge, v. 176, n. 5, p. 1026-1039, 2019. DOI: 10.1016/j.cell.2018.12.028. Disponível em: https://www.sciencedirect.com/ science/article/pii/S0092867418316428. Acesso em: 29 jul. 2020.

WANG, S.-F. et al. Antibody-dependent SARS coronavirus infection is mediated by antibodies against spike proteins. Biochemical and Biophysical Research Communications, New York, v. 451, n. 2, p. 208-214, 2014. DOI: 10.1016/j. bbrc.2014.07.090. Disponível em: https://www.sciencedirect.com/science/article/ pii/S0006291X14013321?via\%3Dihub. Acesso em: 29 jun. 2020.

WEN, W. et al. Immune cell profiling of COVID-19 patients in the recovery stage by single-cell sequencing. Cell Discovery, London, v. 6, n. 31, p. 1-18, 2020. DOI: https://doi.org/10.1038/s41421-020-0168-9. Disponível em: https://www. nature.com/articles/s41421-020-0168-9. Acesso em: 29 jun. 2020.

WÖLFEL, R. et al. Virological assessment of hospitalized patients with COVID2019. Nature, London, v. 581, p. 465-469, 2020. DOI: 10.1038/s41586-0202196-x. Disponível em: https://www.nature.com/articles/s41586-020-2196-x. Acesso em: 29 jun. 2020.

WU, F. et al. Neutralizing antibody responses to SARS-CoV-2 in a COVID-19 recovered patient cohort and their implications. MedRxiv, [s. I.], 2020. DOI: https:// doi.org/10.1101/2020.03.30.20047365. Disponível em: https://www.medrxiv.org/ content/10.1101/2020.03.30.20047365v2. Acesso em: 29 jun. 2020. 
XIAO, A. T.; GAO, C.; ZHANG, S. Profile of specific antibodies to SARS-CoV-2: The first report. Journal of Infection, London, v. 81, n. 1, p. 147-178, 2020. DOI: 10.1016/j.jinf.2020.03.012. Disponível em: https://www.journalofinfection. com/article/S0163-4453(20)30138-9/fulltext. Acesso em: 29 jun. 2020.

YANG, X. et al. Clinical course and outcomes of critically ill patients with SARS-CoV-2 pneumonia in Wuhan, China: a single-centered, retrospective, observational study. Lancet Respiratory Medicine, Kidlington, v. 8, n. 5, p. 475-481, 2020. DOI: https://doi.org/10.1016/S2213-2600(20)30079-5. Disponível em: https://www.thelancet.com/journals/lanres/article/PIIS2213-2600(20)30079-5/ fulltext. Acesso em: 29 jun. 2020.

YASUI, F. et al. Phagocytic cells contribute to the antibody-mediated elimination of pulmonary-infected SARS coronavirus. Virology, New York, v. 454-455, n. 1, p. 157-168, 2014. DOI: 10.1016/j.virol.2014.02.005. Disponível em: https:// linkinghub.elsevier.com/retrieve/pii/S004268221400049X. Acesso em: 29 jun. 2020.

YASUI, F. et al. Prior Immunization with Severe Acute Respiratory Syndrome (SARS)-Associated Coronavirus (SARS-CoV) Nucleocapsid Protein Causes Severe Pneumonia in Mice Infected with SARS-CoV. Journal of Immunology, Baltimore, v. 181, n. 9, p. 6337-6348, 2008. DOI: 10.4049/jimmunol.181.9.6337. Disponível em: https://www.jimmunol.org/content/181/9/6337. Acesso em: 29 jun. 2020.

YONGCHEN, Z. et al. Different longitudinal patterns of nucleic acid and serology testing results based on disease severity of COVID-19 patients. Emerging Microbes \& Infection, New York, v. 9, n. 1, p. 833-836, 2020. DOI: 10.1080/22221751.2020.1756699. Disponível em: https://www.tandfonline. com/doi/full/10.1080/22221751.2020.1756699. Acesso em: 29 jul. 2020.

ZHANG, B. et al. Immune phenotyping based on neutrophil-to-lymphocyte ratio and IgG predicts disease severity and outcome for patients with COVID-19. MedRxiv, [s. I.], 2020. Disponível em: https://www.medrxiv.org/ content/10.1101/2020.03.12.20035048v1. Acesso em: 29 jun. 2020.

ZHAO, J. et al. Antibody Responses to SARS-CoV-2 in Patients of Novel Coronavirus Disease. Clinical Infectious Diseases, Chicago, 2020. DOI: 10.1093/cid/ ciaa344. Disponível em: https://academic.oup.com/cid/article/doi/10.1093/cid/ ciaa344/5812996. Acesso em: 29 jun. 2020. 